\title{
Relazione impresa-territorio e modelli predittivi del default d'impresa. Primi risultati di una analisi statistica sulle piccole imprese italiane
}

\author{
FRANCESCO CIAMPI NICCOLÒ GORDINI ${ }^{* *}$
}

\begin{abstract}
Obiettivo del paper: Verificare se l'utilizzo di variabili qualitative inerenti il territorio elo la relazione impresa-territorio consente di migliorare l'efficacia dei modelli predittivi del default della piccola impresa.

Metodologia: Abbiamo applicato la regressione logistica ad un campione di 736 piccole imprese italiane ed abbiamo elaborato quattro diversi modelli predittivi del default d'impresa. Abbiamo poi testato l'efficacia previsionale di tali modelli su un campione di conferma.

Risultati: Le variabili relative al territorio di insediamento e, ancor di più, quelle relative alla relazione impresa-territorio consentono di migliorare l'efficacia previsionale dei modelli predittivi. Il potenziale predittivo di tali variabili risulta particolarmente rilevante per le imprese appartenenti alle classi dimensionali inferiori.

Limiti della ricerca: I dati relativi alle variabili qualitative sono influenzati dalle percezioni soggettive del management delle imprese analizzate. Non vengono impiegate variabili qualitative ulteriori, quali quelle inerenti le strategie competitive adottate, le competenze del management, gli attributi inter-organizzativi, ecc. Non vengono impiegate variabili quantitative relative all'andamento della relazione impresa-sistema finanziario.

Implicazioni pratiche: Il lavoro suggerisce l'opportunità, per gli istituti finanziari, di integrare le variabili qualitative relative al territorio e, soprattutto, quelle relative alla relazione impresa-territorio nei processi di elaborazione dei modelli di predizione del default dei propri clienti. Il rating costruito utilizzando congiuntamente tali categorie di variabili consentirebbe, in particolare, di limitare $i$ comportamenti orientati ad un eccessivo razionamento del credito.

Originalità del lavoro: Non risultano in letteratura precedenti studi sul contributo che le variabili territoriali possono fornire alla previsione del default d'impresa.
\end{abstract}

Parole chiave: modelli per la predizione del default d'impresa; rating; relazione impresaterritorio; consonanza

\footnotetext{
Associato di Economia e Gestione delle Imprese - Università degli Studi di Firenze e-mail: francesco.ciampi@unifi.it

** Ricercatore di Economia e Gestione delle Imprese - Università degli Studi di MilanoBicocca

e-mail: niccolo.gordini@unimib.it
} 
Purpose of the paper: To test whether the qualitative variables regarding the territory and/or the firm-territory relationship can improve the accuracy rates of small enterprise business failure prediction models.

Methodology: We applied logistic regression to a sample of 736 Italian small enterprise and built 4 different business failure prediction models. We then tested the accuracy rates obtained with these models against a holdout sample.

Findings: Including territory qualitative variables and, most importantly, those regarding the relationships between firms and their territories, significantly improves the accuracy rates of default prediction models. This improvement is most marked in the case of smaller firms.

Research limits: The qualitative variables data collected are affected by subjective judgments made by managers in the firms studied. In addition, no other qualitative variables (such those regarding competitive strategies, or managerial skills, or inter-organizational characteristics) were included, nor were quantitative variables regarding the relationships between firms and financial institutions.

Practical implications: The study suggests that financial institutions should include territory qualitative variables, and, above all, qualitative variables regarding the firmterritory relationship, when constructing business default prediction models. Including this type of variable could reduce the tendency to place unnecessary restrictions on credit.

Originality of the paper: In the field of business failure prediction modeling no research has been done into whether it would be useful to adopt qualitative variables regarding the firm-territory relationship. It is a unexplored area.

Key words: business failure prediction modeling; rating; firm-territory relationships; consonance

\section{Introduzione}

La maggior parte degli studi aventi ad oggetto la modellizzazione dei processi di predizione del default d'impresa si caratterizza per l'impiego, quali variabili indipendenti, degli indici di bilancio.

L'utilizzo dei soli ratios economico-finanziari presenta tuttavia limiti rilevanti quando le previsioni da formulare hanno ad oggetto la Piccola Impresa (PI). Innanzitutto l'informativa di bilancio prodotta dalla PI è di norma meno articolata, approfondita ed affidabile rispetto a quella delle imprese di maggiori dimensioni. Inoltre è tipico che nelle PI proprietario e manager coincidano, il vertice imprenditoriale tenda a prendere le proprie decisioni in maniera fortemente accentrata, la struttura organizzativa sia elementare, con la conseguenza che $i$ mutamenti di struttura e di strategia avvengono spesso velocemente e verso direzioni poco prevedibili sulla base della semplice estrapolazione degli andamenti economico-finanziari passati (Ciampi, 1994). La PI si trova poi a dover affrontare condizionamenti esterni (strategici ed operativi) più intensi, variabili e differenziati rispetto a quanto avviene per l'impresa di media o grande dimensione: un anno, così, l'utile di esercizio potrebbe risultare inferiore a quello fisiologico perché più valore è stato distribuito ai clienti (ad esempio perché il cliente principale, facendo leva sul suo potere contrattuale, ha costretto l'impresa a comprimere il prezzo unitario di vendita), oppure ai dipendenti (perché si è deciso di incrementare lo stipendio di 
alcuni dipendenti al fine di evitare un loro passaggio ad un'impresa concorrente), o ad un fornitore (perché incrementi dei prezzi unitari di acquisto sono stati "imposti" dall'unico fornitore di un semilavorato essenziale o di un servizio non rinunciabile). Dimensione aziendale più contenuta significa infine valori assoluti delle grandezze (anche contabili) più contenuti e, quindi, ratios più sensibili a variazioni anche piccole delle grandezze (tanto che, sotto certi livelli dimensionali, alcuni indicatori perdono quasi completamente di efficacia dimostrativa).

È inoltre oggi assunto condiviso che le possibilità di sopravvivenza dell'impresa siano strettamente dipendenti dalla sua capacità di localizzare i moduli della catena del valore in contesti territoriali nei quali sono presenti ed accessibili le risorse e le competenze necessarie per il suo successo competitivo; oltre che dalla sua capacità di sviluppare con tali contesti relazioni di consonanza (Barile, 2009; Golinelli, 2010, 2011; Golinelli et al., 2002), che consentano la effettiva ed armonica condivisione e combinazione di tali risorse e competenze (Cooke, 2002; De Maio e Maggiore, 1992; Moran e Ghoshal, 1996; Tsai e Ghoshal, 1998).

Tale assunto, le marcate peculiarità gestionali delle PI ed il minor potenziale predittivo che l'informazione contabile assume per questa categoria di imprese fanno emergere l'esigenza di provare a costruire e testare modelli predittivi del default d'impresa: (1) specificamente progettati per la PI; (2) costruiti utilizzando non solo indici di bilancio ma anche variabili relative alle qualità (tangibili ed intangibili) della relazione impresa-territorio.

Obiettivo del presente lavoro è proprio quello di verificare se l'utilizzo delle variabili relative al territorio ed alla relazione impresa-territorio consente di migliorare l'efficacia dei modelli predittivi del default della piccola impresa.

Il presente lavoro è così articolato: sintetica review della letteratura inerente la modellizzazione dei processi di previsione del default d'impresa, ipotesi della ricerca, descrizione del campione oggetto di analisi (e dei criteri utilizzati per la sua estrazione), esposizione delle variabili indipendenti selezionate e della metodologia statistica utilizzata, esposizione e commento dei risultati, conclusioni finali.

\section{La previsione del default d'impresa: una sintetica review della letteratura}

A partire dal lavoro di Altman (1968) numerose analisi empiriche hanno testato l'utilizzo degli indici di bilancio ai fini dell'elaborazione dei modelli predittivi del default d'impresa. L'analisi discriminante multivariata (Altman, 1968 ${ }^{1}$; Altman,

1 Altman (1968) applica l'analisi discriminante multivariata ad un campione di 66 imprese industriali di medie e grandi dimensioni (33 in default e 33 in bonis) e dimostra che l'utilizzo di indici di bilancio appropriatamente selezionati consente di ottenere, con un anno di anticipo rispetto al default, un tasso di corretta classificazione delle imprese pari al $79 \%$. 
1993; Altman et al., $1977^{2}$; Blum, 1974², Deakin, 1972) e l'analisi logistica (Altman e Saunders, 1996; Ohlson, 19804) rappresentano le tecniche statistiche più frequentemente utilizzate. Studi successivi hanno applicato metodologie diverse quali le reti neurali (Ciampi e Gordini, 2013; Vallini et al., 2009; Zhang et al., 1999), gli algoritmi genetici (Back et al., 1996), gli alberi decisionali (Lee et al., 1996), i modelli MARS (Multivariate Adaptive Regression Splines; Olmeda e Fernandez, 1997), i sistemi del tipo CBF (Case-Based Forecasting; Jo et al., 1997), i modelli applicativi della Rough Set Theory (Greco et al., 1998).

Altri studi hanno testato l'utilizzo di variabili intra-organizzative ulteriori rispetto ai ratios di bilancio. Un primo filone ha analizzato il potenziale predittivo delle variabili inerenti i caratteri del management, quali il grado di competenza gestionale (Haswell e Holmes, 1989), il grado di esperienza pregressa nel settore di attività dell'impresa o anche al di fuori di esso (Larson e Clute, 1979), il livello di istruzione e le competenze in materia di marketing e di finanza (Lussier, 1995), la presenza di legami di parentela con altri imprenditori (Cooper et al., 1991; Lussier, 1995), il grado di anzianità di ruolo (Cooper et al., 1991; Lussier, 1995), il grado di propensione al rischio (Koh, 1996), il need for achievement (Cooper et al., 1991; Koh, 1996), le convinzioni circa il locus of control (Evans e Leighton, 1989), la propensione all'innovazione (Koh, 1996). Von Stein e Ziegler (1984) ad esempio, analizzando un campione di 160 piccole e medie imprese (135 in default e 25 in bonis), dimostrano che l'impiego delle variabili relative al management (livello di istruzione, esperienza pregressa, competenze detenute nelle diverse aree funzionali e grado di propensione al rischio) consente di incrementare significativamente l'efficacia dei modelli previsionali. Un secondo filone di studi ha testato il potenziale predittivo di variabili inerenti la corporate governance quali la dimensione e la composizione del consiglio di amministrazione, la presenza di amministratori indipendenti e la CEO-Duality (Chaganti et al., 1985; Daily e Dalton, 1994).

2 Il tasso di accuratezza previsionale del modello "Zeta Score" di Altman et al., (1977) (sviluppato tramite l'applicazione dell'analisi discriminante multivariata ad un campione di 111 imprese e basato sull'utilizzo di 7 indici di bilancio) è pari al 96\% un anno prima del default ed al $70 \%$ cinque anni prima.

3 Blum (1974) applica l'analisi discriminante multivariata ad un campione costituito da 230 imprese industriali di medie e grandi dimensioni (115 fallite e 115 in bonis) e sviluppa un modello previsionale (fondato sull'utilizzo di indici di bilancio) in grado di stimare correttamente il default/non default nel $95 \%$ dei casi con un anno di anticipo e nell' $80 \%$ dei casi con due anni di anticipo.

4 Ohlson (1980), applicando la regressione logistica ad un campione di 105 imprese industriali, elabora un modello di previsione del default in grado di ottenere un tasso di accuratezza previsionale del $96,1 \%$ un anno prima del default e del $95,5 \%$ due anni prima.

5 Jo, Han e Lee (1997) confrontano le reti neurali, l'analisi discriminante multivariata e la tecnica Case Based Reasoning, ottenendo gradi di accuratezza previsionale pari rispettivamente al $83,79 \%$, all' $82,22 \%$ ed all' $81,52 \%$. 
L'analisi del potenziale predittivo delle variabili inerenti la relazione impresaterritorio resta invece un ambito di ricerca ancora completamente inesplorato. Le ipotesi della presente ricerca sono state formulate proprio con l'intento di indagare tale promettente ambito, con particolare riferimento al caso delle imprese di piccole dimensioni.

\section{Ipotesi}

Abbiamo formulato le seguenti ipotesi di ricerca.

Sulla base delle considerazioni sopra esposte e della ampia letteratura che ha approfondito il tema delle relazioni tra variabili territoriali e competitività aziendale $^{6}$, ci aspettiamo che i modelli previsionali costruiti impiegando anche le variabili inerenti il "territorio di insediamento" abbiano una capacità previsionale del default d'impresa più elevata rispetto ai modelli fondati sulle sole variabili economico-finanziarie. Pertanto:

H1A: L'utilizzo congiunto di ratios economico-finanziari e di variabili inerenti il "territorio di insediamento" (Modello 2) consente di ottenere un grado di un'accuratezza previsionale superiore rispetto all'utilizzo dei soli indici di bilancio (Modello 1).

Inoltre ogni impresa si configura quale sistema "unico" di risorse, capacità e competenze, in grado di attivare relazioni "uniche" con il suo territorio di riferimento. Quindi:

H1B: L'utilizzo congiunto di ratios economico-finanziari e di variabili inerenti la "relazione impresa-territorio" (Modello 3) consente di ottenere un grado di accuratezza previsionale superiore rispetto all'utilizzo dei soli indici di bilancio (Modello 1).

H1C: Il modello previsionale costruito utilizzando congiuntamente ratios economico-finanziari, variabili inerenti il "territorio di insediamento" $e$ variabili inerenti la "relazione impresa-territorio" (Modello 4) consente di ottenere un grado di accuratezza previsionale superiore rispetto agli altri tre modelli.

La quasi totalità degli studi aventi ad oggetto la modellizzazione dei processi di predizione del default d'impresa fonda i propri risultati sull'utilizzo di dati raccolti presso campioni di imprese di medie e/o grandi dimensioni (Altman, 1968; Altman, 1993; Blum, 1974), sulla base dell'implicito assunto che i modelli così elaborati possano avere valore predittivo per le imprese di qualsiasi dimensione. Solo un

6 Per una rassegna delle fonti bibliografiche considerate ai fini del presente studio si veda il paragrafo 5 . 
limitato numero di ricerche relativamente recenti ha evidenziato la necessità di sviluppare modelli previsionali diversi per imprese appartenenti a diverse classi dimensionali, ed in particolare di costruire modelli di previsione del default che tengano conto degli specifici caratteri strutturali, gestionali e strategici delle piccole imprese (Altman e Sabato, 2007; Altman et al., 2010; Behr e Guttler, 2007; Carter e Van Auken, 2006; Ciampi e Gordini, 2008; 2009; 2013; Pompe e Bilderbeek, 2005; Saurina e Trucharte, 2004; Vallini et al., 2009; Vallini et al., 2008, 2009). Una ulteriore ipotesi della nostra ricerca inerisce quindi l'incremento del grado di accuratezza previsionale ottenibile tarando la funzione decisionale sulla base di sotto-campioni di imprese sufficientemente omogenei dal punto di vista della dimensione aziendale.

In particolare:

H2: Quando la regressione logistica è applicata per singola classe dimensionale tutti $i$ modelli forniscono un grado di accuratezza previsionale significativamente più elevato rispetto a quello ottenuto elaborando la funzione decisionale su base aggregata.

\section{Data Set}

Il campione da noi analizzato è costituito da 4.277 piccole imprese estratte dagli archivi Cerved (tali archivi contengono l'informazione contabile, raccolta attraverso la rete delle Camere di Commercio, relativa all'universo delle società di capitale italiane).

Quale evento di default abbiamo individuato l'avvio di una procedura concorsuale (fallimento, concordato preventivo, amministrazione controllata). Si tratta di una soluzione più restrittiva (default conclamato) rispetto a quella di norma utilizzata nell'ambito della modellistica bancaria, che identifica il default con l'emergere di una situazione di insolvenza di fatto del debitore, non autonomamente risolvibile e tale da provocare una potenziale perdita del credito concesso.

Il campione delle imprese in default è costituito da tutte le imprese italiane incluse negli archivi Cerved, operanti nei rami industria, costruzioni e servizi (con esclusione, quindi, delle società immobiliari e di quelle finanziarie), entrate in stato di default nell'anno 2008 e che presentavano nel 2005 un bilancio di esercizio regolarmente depositato ed un fatturato inferiore a 5 milioni di euro. La numerosità di tale campione è risultata pari a 2.234.

L'insieme delle imprese non in default è stato selezionato tramite campionamento stratificato a partire dall'universo delle imprese italiane incluse negli archivi Cerved operanti nei rami industria, costruzioni e servizi, per le quali non era stata avviata una procedura concorsuale alla fine del 2008 e che presentavano nel 2005 un bilancio di esercizio regolarmente depositato ed un 
fatturato inferiore a 5 milioni di euro ${ }^{7}$. Per la selezione di tale campione abbiamo utilizzato le seguenti variabili: dimensione d'impresa (articolata sulla base delle quattro classi di fatturato $^{8}$ individuate nella Tabella 1), area geografica d'insediamento (Nord, Centro e Sud) e ramo di attività economica (ramo industriale, ramo costruzioni, ramo dei servizi) ${ }^{9}$. Sono state così estratte 2.043 imprese.

Tab. 1: Il Data Set (valori percentuali)

\begin{tabular}{|l|r|r|}
\hline & $\begin{array}{c}\text { Imprese in } \\
\text { default }\end{array}$ & $\begin{array}{c}\text { Imprese non in } \\
\text { default }\end{array}$ \\
\hline Area Geografica & & \\
\hline Nord & 36,1 & 39,8 \\
\hline Centro & 35,3 & 36,2 \\
\hline Sud & 28,6 & 24,0 \\
\hline Settore & & \\
\hline Industria & 42,6 & 41,1 \\
\hline Costruzioni & 11,9 & 11,4 \\
\hline Servizi & 45,5 & 47,5 \\
\hline Dimensione (Fatturato in Milioni di Euro) & & \\
\hline Dimensione 1 (<0,5) & 40,4 & 43,6 \\
\hline Dimensione 2 (0,5-1) & 24,4 & 24,1 \\
\hline Dimensione 3 (1-2,5) & 24,1 & 22,0 \\
\hline Dimensione 4 (2,5-5) & & 10,3 \\
\hline Sesso della persona rispondente (Valori \%) & 92,1 & 93,4 \\
\hline Maschi & 7,9 & 6,6 \\
\hline Femmine & 58 & 56 \\
\hline Età Media delle persone rispondenti & 338 & 398 \\
\hline Numerosità Totale & & \\
\hline
\end{tabular}

Fonte: ns. elaborazione

I ratios economico-finanziari (riportati nella successiva Tabella 2) sono stati calcolati utilizzando i dati dei bilanci relativi all'esercizio 2005 estratti degli archivi Cerved.

Al fine di rilevare le variabili relative al "territorio di insediamento" ed alla "relazione impresa-territorio" (elencate nelle successive Tabella 3 e 4) abbiamo elaborato un questionario iniziale e lo abbiamo sottoposto a due pre-test, che ci hanno consentito di migliorarne la qualità, modificando sia la lunghezza che la

7 Da un punto di vista computazionale affiancare un universo (le imprese fallite) a un campione (quelle non fallite) non comporta controindicazioni particolari, se non la perdita di significatività dell'intercetta nella stima logistica.

8 La dimensione delle imprese è stata determinata sulla base del valore del fatturato realizzato nell'esercizio 2005. Le classi dimensionali sono state determinate sulla base dei quartili della distribuzione dimensionale del campione delle imprese in default.

9 L'estrazione del campione delle imprese non in default è stata effettuata in modo da ottenere distribuzioni dimensionali, geografiche e settoriali il più possibile simili a quelle fatte rilevare dal campione delle imprese in default. 
forma della maggioranza delle domande (Lussier, 1995). Il questionario definitivo è risultato composto da 35 domande ordinate casualmente ${ }^{10}$. Tutte le variabili sono state misurate tramite scala di Likert (valori compresi tra 1 e 5). Il questionario definitivo è stato somministrato via mail o via posta ad un componente del consiglio di amministrazione o al direttore generale in carica nell'anno 2005 presso ciascuna delle 4.277 piccole imprese del nostro campione. Per incrementare il tasso di risposta abbiamo implementato una attività di follow up finalizzata a sollecitare le imprese inizialmente non rispondenti. Per la somministrazione e la raccolta dei questionari e per l'inserimento dei dati raccolti all'interno del database ci siamo avvalsi della collaborazione di quattro studenti della Facoltà di Economia dell'Università di Firenze. 736 imprese (338 in default e 398 in bonis) hanno risposto in maniera completa al questionario (tasso di risposta del 17,2\%).

Infine abbiamo suddiviso il nostro campione (formato dalle 736 imprese che hanno risposto al questionario) in due sotto-campioni, costruiti tramite campionamento casuale stratificato realizzato sulla base dei tre criteri di classificazione sopra citati (dimensione, area geografica, ramo di attività economica). Il primo sotto-campione (training sample), composto da 460 imprese (214 in default e 246 in bonis), è stato utilizzato per costruire i modelli previsionali; il secondo (holdout sample), costituito da 276 imprese (124 in default e 152 in bonis), è stato utilizzato per testare l'efficacia previsionale dei modelli elaborati. La Tabella 1 espone la struttura del campione complessivo (736 unità), oltre alla distribuzione per sesso e l'età media delle persone che hanno risposto al questionario. I casi di non default si caratterizzano per una frequenza (in termini relativi) più elevata di imprese appartenenti alla prima classe dimensionale, di imprese operanti nel Nord del Paese, di imprese attive nel ramo servizi.

\section{Selezione delle variabili indipendenti}

I 21 indici di bilancio impiegati ai fini della nostra analisi (cfr. Tabella 2) sono stati selezionati sulla base delle principali evidenze emerse in letteratura (Altman, 1968, 1993; Altman e Sabato, 2007; Blum, 1974; Crouhy et al., 2001; Edmister, 1972).

10 Il contenuto del questionario è articolato come segue:

- dati anagrafici dell'impresa (ivi inclusi il fatturato, il numero di addetti ed il settore di attività economica);

- dati anagrafici della persona intervistata;

- 29 quesiti finalizzati a misurare (tramite scala di Likert) ciascuna delle 29 variabili riportate nelle Tabelle 3 e 4;

- 6 quesiti "di controllo". 
Tab. 2: Il set iniziale degli indicatori economico-finanziari (valori medi per gruppo)

\begin{tabular}{|l|c|c|}
\hline & $\begin{array}{c}\text { Imprese in } \\
\text { default }\end{array}$ & $\begin{array}{c}\text { Imprese non in } \\
\text { default }\end{array}$ \\
\hline ROE & $-4,2$ & 5,6 \\
\hline ROI & 1,0 & 5,4 \\
\hline ROS & 1,3 & 4,4 \\
\hline ROA & 0,7 & 4,8 \\
\hline Valore aggiunto/Fatturato & 13,4 & 22,2 \\
\hline Ebitda/Fatturato & 2,4 & 7,91 \\
\hline Ebitda/Cash flow & 63,4 & 98,2 \\
\hline Interessi Passivi/Fatturato & 5,7 & 3,8 \\
\hline Interessi Passivi/Ebitda & 63,7 & 36,2 \\
\hline Fatturato/Numero di addetti & 97,8 & 188,6 \\
\hline Valore Aggiunto/Numero di addetti & 29,9 & 54,3 \\
\hline Attivo immobilizzato/Numero di addetti & 37,9 & 29,3 \\
\hline Cash flow/Debiti totali & 2,4 & 7,9 \\
\hline Cash Flow/Fatturato & 1,5 & 3,6 \\
\hline Interessi Passivi/Debiti Bancari & 9,6 & 7,90 \\
\hline Debiti Bancari/Fatturato & 0,79 & 0,45 \\
\hline Debiti Totali/(Debiti Totali + Equity) & 74,5 & 55,4 \\
\hline Debiti totali/Ebitda & 766,7 & 425,2 \\
\hline Equity/Immobilizzazioni Materiali & 46,2 & 72,4 \\
\hline Current Ratio & 0,799 & 1,123 \\
\hline Fatturato/Capitale Investito & 0,788 & \\
\hline
\end{tabular}

Fonte: ns. elaborazione

Le Tabelle 3 e 4 descrivono le variabili "territoriali" da noi selezionate traendo spunto dalla ampia letteratura aziendale ed economica che, con diverse prospettive (da quella dei distretti marshalliani, a quella dei cluster porteriani, a quella dei sistemi regionali di innovazione), ha approfondito le relazioni tra caratteri dei contesti territoriali e competitività aziendale (nell'ultima colonna sono indicate le principali fonti bibliografiche considerate ai fini della individuazione e definizione di ciascuna variabile). 
Tab. 3: Il set iniziale delle variabili relative ai caratteri del territorio di insediamento dellimpresa

\begin{tabular}{|c|c|c|}
\hline VARIABILI & DESCRIZIONE & $\begin{array}{l}\text { ALCUNI RIFERIMENTI BIBLIOGRAFICI } \\
\text { (IN ORDINE ALFABETICO) }\end{array}$ \\
\hline \multicolumn{3}{|c|}{ Caratteri tangibili } \\
\hline $\begin{array}{l}\text { Posizione } \\
\text { geografica }\end{array}$ & $\begin{array}{l}\text { Grado di attrattività del territorio in } \\
\text { termini di posizione geografica* }\end{array}$ & \multirow{5}{*}{$\begin{array}{l}\text { Baccarani e Golinelli (1993); Becattini (1979, 1989, 1990); Brusco } \\
\text { (1982); Krugman (1991a, 1991b); Krugman (1995); Marshall (1919, } \\
\text { 1925); Piore e Sabel (1984); Porter (1990, 1998); Varaldo (2006); } \\
\text { Varaldo e Ferrucci (1996) }\end{array}$} \\
\hline $\begin{array}{l}\text { Assetto } \\
\text { morfologico }\end{array}$ & $\begin{array}{l}\text { Grado di attrattività del territorio in } \\
\text { termini di assetto morfologico* }\end{array}$ & \\
\hline $\begin{array}{l}\text { Dotazione di } \\
\text { infrastrutture }\end{array}$ & $\begin{array}{l}\text { Grado di attrattività del territorio in } \\
\text { termini di infrastrutture disponibili * }\end{array}$ & \\
\hline Risorse naturali & $\begin{array}{l}\text { Grado di attrattività del territorio in } \\
\text { termini di risorse naturali disponibili * }\end{array}$ & \\
\hline $\begin{array}{l}\text { Articolazione del } \\
\text { tessuto produttivo }\end{array}$ & $\begin{array}{l}\text { Grado di attrattività del territorio in } \\
\text { termini di articolazione di attività } \\
\text { produttive svolte localmente * }\end{array}$ & \\
\hline $\begin{array}{l}\text { Articolazione } \\
\text { "fisica" del sistema } \\
\text { finanziario }\end{array}$ & $\begin{array}{l}\text { Grado di attrattività del territorio in } \\
\text { termini di presenza e diffusione } \\
\text { spaziale di istituti bancari ed altre } \\
\text { istituzioni finanziarie * }\end{array}$ & $\begin{array}{l}\text { Alessandrini et al., (2007); Baffigi et al. (2000); Jimenez et al. (2007); } \\
\text { Signorini (2000); Ughetto (2006) }\end{array}$ \\
\hline \multicolumn{3}{|c|}{ Caratteri intangibili } \\
\hline $\begin{array}{l}\text { Capitale sociale e } \\
\text { fiducia }\end{array}$ & $\begin{array}{l}\text { Grado di attrattività del territorio come } \\
\text { sistema di relazioni sociali fondate } \\
\text { sulla fiducia tra gli attori * }\end{array}$ & \multirow{3}{*}{$\begin{array}{l}\text { Baccarani e Golinelli (1993); Becattini (1979, 1989, 1990); Belussi e } \\
\text { Pilotti (2008); Brusco (1982, 1999); Capello (2002); Coleman (1988, } \\
\text { 1990); Cooke (2002); Dei Ottati (1994, 2003); Denicolai et al., (2010); } \\
\text { Dyer e Singh (1998); Gargiulo e Benassi (2000); Ghoshal e Barlett } \\
\text { (1994); Golinelli (2002); Grandinetti e Tabacco (2003); Granovetter } \\
\text { (1985); Gulati et al. (2000); Lazerson e Lorenzoni (1999); Leiponen e } \\
\text { Byma (2009); Lipparini e Lorenzoni (1996); Lorenz (1992); Lundvall } \\
\text { (1992); Malecki (1995, 2000); Maskell e Malmberg (2007); Mustilli et } \\
\text { al., (2011); Nahapiet e Ghoshal (1998); Nooteboom (2004); Piore e } \\
\text { Sabel (1984); Putman (1993, 1995); Tsai e Ghoshal (1998); Uzzi } \\
\text { (1996, 1997); Varaldo (2006); Varaldo e Ferrucci (1996) }\end{array}$} \\
\hline Cultura & $\begin{array}{l}\text { Qualità del territorio in termini di } \\
\text { sistema culturale locale (storia, } \\
\text { tradizioni e saperi di un luogo) * }\end{array}$ & \\
\hline Sistema di valori & $\begin{array}{l}\text { Grado di attrattività del territorio come } \\
\text { sistema di valori consolidati e condivisi }\end{array}$ & \\
\hline Innovazione & $\begin{array}{l}\text { II contesto territoriale favorisce/limita i } \\
\text { processi di innovazione }{ }^{* *}\end{array}$ & $\begin{array}{l}\text { Acs et al. (2002); Ahuja (2000); Asheim e Coenen (2005); Asheim e } \\
\text { Gertler (2005); Asheim e Isaksen (2002); Autio (1998); Belussi et al., } \\
\text { (2003); Bortoluzzi e Tracogna (2011); Boschma (2005); Bottinelli e } \\
\text { Pavione (2010); Braczyck e Cooke (1998); Breschi e Malerba (2005); } \\
\text { Buesa et al., (2010); Camuffo e Grandinetti (2006); Crevosier e } \\
\text { Camagni (2000); Cooke (2001); Cooke et al. (1997); Cooke e Morgan } \\
\text { (1998); Corò e Micelli (2006, 2007); Corò e Rullani (1998); Dagnino, et } \\
\text { al., (2011); Doloreux e Parto (2005); Lundvall (1992); Maillat e Perrin } \\
\text { (1992); Maillat et al., (1993); Micelli e Chiarvesio (2003); Mustilli et al., } \\
\text { (2011); Porter (1998); Robertson et al. (2009); Rolfo (1996); Santoni e } \\
\text { Zanni (2011); Todtling e Trippl (2005); Velo (2007, 2011) }\end{array}$ \\
\hline $\begin{array}{l}\text { Internazionaliz- } \\
\text { zazione }\end{array}$ & $\begin{array}{l}\text { II contesto territoriale favorisce/limita i } \\
\text { processi di internazionalizzazione }{ }^{* *}\end{array}$ & $\begin{array}{l}\text { Becattini e Rullani (1993); Chiarvesio e Micelli (2007); Corò e Micelli } \\
\text { (2006); Micelli e Chiarvesio (2003); Rullani (2006); Varaldo (2006) }\end{array}$ \\
\hline $\begin{array}{l}\text { Sistema di risorse } \\
\text { e competenze } \\
\text { radicate e } \\
\text { condivise }\end{array}$ & $\begin{array}{l}\text { Grado di attrattività del territorio come } \\
\text { sistema di risorse e competenze } \\
\text { radicate e condivise * }\end{array}$ & $\begin{array}{l}\text { Baccarani e Golinelli (1993); Becattini (1979, 1990, 2000); Bellandi } \\
\text { (2009); Belussi e Pilotti (2008); Boari e Lipparini (1999); Brusco (1982, } \\
\text { 1989); Camagni (1991); Foss (1996); Glaeser et al. (1992); Golinelli } \\
\text { (2002); Hernderson (1996); Marshall (1919, 1925); Maskell e } \\
\text { Malmberg (2007); Porter (1990, 1998); Quadrio et al. (2002); Rullani } \\
\text { (2003); Saxenian (1994); Varaldo e Ferrucci (1996) }\end{array}$ \\
\hline $\begin{array}{l}\text { Competitività del } \\
\text { sistema finanziario }\end{array}$ & $\begin{array}{l}\text { Grado di attrattività del territorio in } \\
\text { termini di competitività del sistema } \\
\text { finanziario locale * }\end{array}$ & $\begin{array}{l}\text { Alessandrini et al. (2006); Liberti e Mian (2006); Lugaresi e Rotondi } \\
\text { (2007); Pagnini (2000); Rotondi (2005); Russo e Rossi (1999) }\end{array}$ \\
\hline Reputazione & $\begin{array}{l}\text { Grado di attrattività del territorio in } \\
\text { termini di reputazione * }\end{array}$ & $\begin{array}{l}\text { Becattini (1990); Brady (2002); Cairoli (2011); Denicolai et al. (2010); } \\
\text { Ely e Valimaki (2003); Engelmann e Fischbacher (2003); Ferguson et } \\
\text { al. (2000); Molina-Morales e Martinez-Fernández (2003); Nooteboom } \\
\text { (2002); Sako (1991); Smitka (1991) }\end{array}$ \\
\hline Qualità della vita & $\begin{array}{l}\text { Grado di attrattività del territorio in } \\
\text { termini di benessere e qualità della } \\
\text { vita* }\end{array}$ & Putman (1993) \\
\hline $\begin{array}{l}\text { Sistema delle } \\
\text { Istituzioni Locali }\end{array}$ & $\begin{array}{l}\text { Contributo delle Istituzioni Locali (enti } \\
\text { locali, università, centri di ricerca, } \\
\text { associazioni di categoria, ecc.) allo } \\
\text { sviluppo imprenditoriale del territorio * }\end{array}$ & $\begin{array}{l}\text { Asheim e Isaksen (2002); Bozeman (2000); Camuffo e Grandinetti } \\
\text { (2006); Cooke et al. (1997); Cooke e Morgan (1998); Doloreux e Parto } \\
\text { (2005); Dosi et al. (1998); Galaskiewicz (1985); Lundvall (1992); } \\
\text { McEvily e Zaheer (1999); Mele (2011); Molina-Morales e Martinez- } \\
\text { Fernández (2003); Rullani (2006); Schillaci et al. (2009); Suchman } \\
\text { (1994) }\end{array}$ \\
\hline
\end{tabular}

Note: * Variabile misurata tramite scala di Likert: 1 nullo/a; 2 minimo/a; 3 discreto/a; 4 elevato/a; 5 molto elevato/a.

** Variabile misurata tramite scala di Likert: 1 limita; 2 limita leggermente; 3 è neutro; 4 favorisce leggermente; 5 favorisce.

Fonte: ns. elaborazione 
Tab. 4: Il set iniziale delle variabili relative ai caratteri della relazione impresa-territorio di insediamento

\begin{tabular}{|c|c|c|}
\hline VARIABILI & DESCRIZIONE & ALCUNI RIFERIMENTI BIBLIOGRAFICI (IN ORDINE ALFABETICO) \\
\hline $\begin{array}{l}\text { Grado di } \\
\text { radicamento } \\
\text { territoriale }\end{array}$ & $\begin{array}{l}\text { Grado di radicamento } \\
\text { dell'impresa sul territorio ** }\end{array}$ & $\begin{array}{l}\text { Becattini (1990); Geringer et al. (2000); Hundley e Jacobson (1998); } \\
\text { Marshall (1919); Molina-Morales e Martinez-Fernàndez (2003) }\end{array}$ \\
\hline $\begin{array}{l}\text { Risorse del } \\
\text { territorio ed } \\
\text { immagine } \\
\text { aziendale }\end{array}$ & $\begin{array}{l}\text { Impatto delle } \begin{array}{l}\text { risorse del } \\
\text { territorio } \\
\text { sull'immagine } \\
\text { aziendale * }\end{array} \\
\end{array}$ & $\begin{array}{l}\text { Becattini (1990); Brady (2002); Cairoli (2011); Denicolai et al. (2010); } \\
\text { Ely e Valimaki (2003); Engelmann e Fischbacher (2003); Ferguson et al. } \\
\text { (2000); Molina-Morales e Martinez-Fernández (2003); Nooteboom } \\
\text { (2002); Sako (1991); Smitka (1991) }\end{array}$ \\
\hline $\begin{array}{l}\text { Risorse del } \\
\text { territorio e } \\
\text { capacità di } \\
\text { vendita }\end{array}$ & $\begin{array}{l}\text { Impatto delle risorse del } \\
\text { territorio sulle capacità di } \\
\text { vendita * }\end{array}$ & \multirow{5}{*}{$\begin{array}{l}\text { Baccarani e Golinelli (1993); Becattini (1979, 1990, 2000); Bellandi } \\
\text { (2009); Belussi e Pilotti (2008); Boari e Lipparini (1999); Brusco (1982, } \\
\text { 1989); Camagni (1991); Foss (1996); Glaeser et al. (1992); Golinelli } \\
\text { (2002); Hernderson (1996); Marshall (1919, 1925); Maskell e Malmberg } \\
\text { (2007); Porter (1990, 1998); Quadrio Curzio e Fortis (2002); Rullani } \\
\text { (2003); Saxenian (1994); Varaldo e Ferrucci (1996) }\end{array}$} \\
\hline $\begin{array}{l}\text { Risorse del } \\
\text { territorio e } \\
\text { capacità } \\
\text { logistiche }\end{array}$ & $\begin{array}{l}\text { Impatto delle risorse del } \\
\text { territorio sulle capacità } \\
\text { logistiche * }\end{array}$ & \\
\hline $\begin{array}{l}\text { Risorse del } \\
\text { territorio e } \\
\text { capacità } \\
\text { strategiche }\end{array}$ & $\begin{array}{l}\text { Impatto delle risorse del } \\
\text { territorio sulle capacità } \\
\text { strategiche* }\end{array}$ & \\
\hline $\begin{array}{l}\text { Risorse del } \\
\text { territorio e qualità } \\
\text { delle risorse } \\
\text { umane }\end{array}$ & $\begin{array}{l}\text { Impatto delle risorse del } \\
\text { territorio sulla qualità delle } \\
\text { risorse umane * }\end{array}$ & \\
\hline $\begin{array}{l}\text { Risorse del } \\
\text { territorio e } \\
\text { capacità di } \\
\text { controllo dei costi }\end{array}$ & $\begin{array}{l}\text { Impatto delle risorse del } \\
\text { territorio sulle capacità di } \\
\text { controllo dei costi * }\end{array}$ & \\
\hline $\begin{array}{l}\text { Risorse del } \\
\text { territorio, struttura } \\
\text { intra-organiz- } \\
\text { zativa ed } \\
\text { architettura inter- } \\
\text { organizzativa }\end{array}$ & $\begin{array}{l}\text { Impatto delle risorse del } \\
\text { territorio sui caratteri della } \\
\text { struttura intra-organizzativa } \\
\text { e sugli attributi della } \\
\text { architettura } \\
\text { organizzativa * }\end{array}$ & $\begin{array}{l}\text { Baccarani e Golinelli (1993); Becattini (1979, 1989, 1990); Belussi e } \\
\text { Pilotti (2008); Brusco (1982, 1999); Capello (2002); Coleman (1988, } \\
\text { 1990); Cooke (2002); Dei Ottati (1994, 2003); Denicolai et al., (2010); } \\
\text { Dyer e Singh (1998); Gargiulo e Benassi (2000); Ferri et al., (2009); } \\
\text { Ghoshal e Barlett (1994); Golinelli (2002); Grandinetti e Tabacco (2003); } \\
\text { Granovetter (1985); Gulati (1998); Gulati et al. (2000); Lavie (2006); } \\
\text { Lazerson e Lorenzoni (1999); Leiponen e Byma (2009); Lipparini e } \\
\text { Lorenzoni (1996); Lorenz (1992); Lundvall (1992); Malecki (1995, 2000); } \\
\text { Maskell e Malmberg (2007); Mustilli et al., (2011); Nahapiet e Ghoshal } \\
\text { (1998); Nooteboom (2004); Piore e Sabel (1984); Putman (1993, 1995); } \\
\text { Sobrero e Roberts (2001); Tsai e Ghoshal (1998); Uzzi (1996, 1997); } \\
\text { Varaldo (2006); Varaldo e Ferrucci (1996) }\end{array}$ \\
\hline $\begin{array}{l}\text { Risorse del } \\
\text { territorio e } \\
\text { capacità } \\
\text { tecnologiche }\end{array}$ & $\begin{array}{l}\text { Impatto delle risorse del } \\
\text { territorio sulle capacità } \\
\text { tecnologiche * }\end{array}$ & \multirow{3}{*}{$\begin{array}{l}\text { Acs et al. (2002); Ahuja (2000); Asheim e Coenen (2005); Asheim e } \\
\text { Gertler (2005); Asheim e Isaksen (2002); Autio (1998); Belussi et al., } \\
\text { (2003); Bortoluzzi e Tracogna (2011); Boschma (2005); Bottinelli e } \\
\text { Pavione (2010); Braczyck e Cooke (1998); Breschi e Malerba (2005); } \\
\text { Buesa et al., (2010); Camuffo e Grandinetti (2006); Crevosier e } \\
\text { Camagni (2000); Cooke (2001); Cooke et al. (1997); Cooke e Morgan } \\
\text { (1998); Corò e Micelli (2006, 2007); Corò e Rullani (1998); Dagnino, et } \\
\text { al., (2011); Doloreux e Parto (2005); Lundvall (1992); Maillat e Perrin } \\
\text { (1992); Maillat et al. (1993); Micelli e Chiarvesio (2003); Mustilli et al., } \\
\text { (2011); Porter (1998); Robertson et al. (2009); Rolfo (1996); Santoni e } \\
\text { Zanni (2011); Todtling e Tripp (2005); Velo (2007, 2011) }\end{array}$} \\
\hline $\begin{array}{l}\text { Risorse del } \\
\text { territorio e } \\
\text { capacità } \\
\text { innovative }\end{array}$ & $\begin{array}{l}\text { Impatto delle risorse del } \\
\text { territorio sulle capacità } \\
\text { innovative * }\end{array}$ & \\
\hline $\begin{array}{l}\text { Risorse del } \\
\text { territorio e qualità } \\
\text { tecnica della } \\
\text { proposta di valore } \\
\text { al cliente }\end{array}$ & $\begin{array}{l}\text { Impatto delle risorse del } \\
\text { territorio sulla qualità tecnica } \\
\text { della proposta di valore al } \\
\text { cliente * }\end{array}$ & \\
\hline $\begin{array}{l}\text { Risorse del } \\
\text { territorio e } \\
\text { capacità } \\
\text { finanziaria }\end{array}$ & $\begin{array}{l}\text { Impatto delle risorse del } \\
\text { territorio sulle capacità } \\
\text { finanziarie * }\end{array}$ & $\begin{array}{l}\text { Alessandrini et al. (2006); Alessandrini et al., (2007); Baffigi et al. } \\
\text { (2000); Jimenez et al. (2007); Liberti e Mian (2006); Lugaresi e Rotondi } \\
\text { (2007); Pagnini (2000); Rotondi (2005); Russo e Rossi (1999, 2001); } \\
\text { Signorini (2000); Ughetto (2006) }\end{array}$ \\
\hline $\begin{array}{l}\text { Contributo } \\
\text { dell'impresa allo } \\
\text { sviluppo del } \\
\text { territorio }\end{array}$ & $\begin{array}{l}\text { Contributo dell'impresa allo } \\
\text { sviluppo economico, sociale } \\
\text { e/o culturale del territorio * }\end{array}$ & Baccarani e Golinelli (2011) \\
\hline
\end{tabular}

Note: * Variabile misurata tramite scala di Likert: 1 negativo; 2 leggermente negativo; 3 nullo; 4 leggermente positivo; 5 positivo.

** Variabile misurata tramite scala di Likert: 1 nullo; 2 minimo; 3 discreto; 4 elevato; 5 molto elevato.

Fonte: ns. elaborazione 
Al fine di selezionare, tra tutte quelle riportate nelle precedenti Tabelle 2, 3 e 4, le variabili caratterizzate da maggiore potenziale predittivo e, contemporaneamente, minor grado di correlazione, abbiamo condotto un'analisi di multicollinearità tramite metodo VIF (Variance Inflation Factor). Le variabili con un valore del VIF superiore a 3 sono state escluse. Abbiamo poi implementato la procedura statistica di selezione delle variabili nota come "Metodo Stepwise". La Tabella 5 mostra l'elenco delle variabili selezionate ai fini della elaborazione dei modelli previsionali.

Tab. 5: Variabili selezionate tramite analisi di multicollinearità e analisi stepwise

\begin{tabular}{|c|c|c|c|c|}
\hline & Modello 1 & Modello 2 & Modello 3 & Modello 4 \\
\hline \multicolumn{5}{|l|}{ RATIOS ECONOMICO-FINANZIARI } \\
\hline ROE & $\mathrm{X}$ & & $\mathrm{X}$ & \\
\hline $\mathrm{ROI}$ & $\mathrm{X}$ & & $\mathrm{X}$ & $\mathrm{X}$ \\
\hline Current Ratio & $\mathrm{X}$ & $\mathrm{X}$ & $\mathrm{X}$ & $\mathrm{X}$ \\
\hline Interessi Passivi/Fatturato & $\mathrm{X}$ & $\mathrm{X}$ & & $\mathrm{X}$ \\
\hline Ebitda/Fatturato & $\mathrm{X}$ & $\mathrm{X}$ & $\mathrm{X}$ & $\mathrm{X}$ \\
\hline Debiti Bancari/Fatturato & & & & $\mathrm{X}$ \\
\hline Valore Aggiunto/Fatturato & & $\mathrm{X}$ & $\mathrm{X}$ & \\
\hline \multicolumn{5}{|l|}{$\begin{array}{l}\text { VARIABILI RELATIVE AI CARATTERI } \\
\text { DEL TERRITORIO DI INSEDIAMENTO } \\
\text { DELL'IMPRESA } \\
\end{array}$} \\
\hline Dotazione di infrastrutture & & & & $\mathrm{X}$ \\
\hline Internazionalizzazione & & $\mathrm{X}$ & & \\
\hline Posizione geografica & & $\mathrm{X}$ & & $\mathrm{X}$ \\
\hline Articolazione del tessuto produttivo & & $\mathrm{X}$ & & $\mathrm{X}$ \\
\hline Capitale sociale e fiducia & & $\mathrm{X}$ & & $\mathrm{X}$ \\
\hline Cultura & & $\mathrm{X}$ & & $\mathrm{X}$ \\
\hline Innovazione & & $\mathrm{X}$ & & $\mathrm{X}$ \\
\hline Sistema di risorse e competenze radicate & & $\mathrm{X}$ & & $\mathrm{X}$ \\
\hline Reputazione & & $\mathrm{X}$ & & \\
\hline \multicolumn{5}{|l|}{$\begin{array}{c}\text { VARIABILI RELATIVE AI CARATTERI } \\
\text { DELLA RELAZIONE IMPRESA-TERRITORIO }\end{array}$} \\
\hline Risorse del territorio e capacità innovative & & & $\mathrm{X}$ & \\
\hline $\begin{array}{c}\text { Risorse del territorio ed architettura (intra- ed inter-) } \\
\text { organizzativa }\end{array}$ & & & $\mathrm{x}$ & $x$ \\
\hline $\begin{array}{c}\text { Risorse del territorio } \mathrm{e} \\
\text { qualità delle risorse umane }\end{array}$ & & & $\mathrm{x}$ & $x$ \\
\hline Risorse del territorio ed immagine aziendale & & & $\mathrm{X}$ & $\mathrm{X}$ \\
\hline Risorse del territorio e capacità strategiche & & & $\mathrm{X}$ & $\mathrm{X}$ \\
\hline Contributo dell'impresa allo sviluppo del territorio & & & $\mathrm{X}$ & $\mathrm{X}$ \\
\hline
\end{tabular}

Fonte: ns. elaborazione

Con riferimento, in particolare, ai caratteri inerenti il contesto territoriale ed a quelli relativi alla relazione impresa-territorio, i nostri risultati confermano alcuni assunti consolidati in letteratura. Relativamente ai caratteri del contesto territoriale, oltre a quelli "fisici" (quali la dotazione infrastrutturale e l'articolazione del tessuto economico di riferimento), influiscono sulle probabilità di default dell'impresa caratteri intangibili quali il sistema locale di risorse e competenze condivise (Foss, 1996), il tessuto culturale del territorio (Lazerson e Lorenzoni, 1999), il capitale sociale locale (Tsai e Ghoshal, 1998). Relativamente ai caratteri della relazione impresa-territorio rilevano: a) l'impatto delle risorse del territorio sulle attività 
intangibili aziendali quali l'immagine e la qualità delle risorse umane (Becattini, 1990); b) l'impatto degli asset territoriali sulle capacità aziendali quali quelle innovative, quelle strategiche e quelle organizzative.

\section{Metodologia}

L'analisi discriminante multivariata è stata per molti anni la tecnica statistica più utilizzata per la costruzione di modelli previsionali del default d'impresa (Altman, 1968; Blum, 1974; Deakin, 1972; Edmister, 1972). Affinché tale metodologia statistica sia efficacemente impiegabile devono risultare rispettate le seguenti condizioni: 1) le variabili di input devono essere distribuite normalmente; 2) le matrici di dispersione di gruppo (matrici di varianza e covarianza) devono essere identiche o molto simili nei due gruppi (Barnes, 1982; Karels e Prakash, 1987).

Poiché le variabili previsionali da noi utilizzate (indici di bilancio) non sono né lineari né normalmente distribuite (Karels e Prakash, 1987; Ohlson, 1980), ai fini della nostra analisi abbiamo utilizzato la regressione logistica (modello Logit), tecnica che ben si adatta alle caratteristiche del problema della previsione del default d'impresa (dove la variabile dipendente è binaria: default/non default), in specie quando l'analisi ha ad oggetto campioni di rilevanti dimensioni (Altman e Saunders, 1996; Ohlson, 1980). Nel modello Logit le variabili indipendenti sono combinate in una funzione lineare che stima il logaritmo del rapporto tra la probabilità che un evento si realizzi e la probabilità che esso non si realizzi ${ }^{11}$. La funzione previsionale assume la tipica seguente forma:

$$
\operatorname{In}[\mathrm{PD} /(1-\mathrm{PD})]=\mathrm{a}+\mathrm{BX}+\mathrm{E}
$$

dove PD è la probabilità di default, $(1$ - PD) è la probabilità di non default, a è la costante, $\mathrm{B}$ è il vettore dei coefficienti delle variabili indipendenti, $\mathrm{X}$ è il vettore delle variabili indipendenti (nel nostro caso un set di indici di bilancio ed un insieme di variabili relative al territorio di insediamento ed alla relazione impresa-territorio), ed E è l'errore.

\section{Risultati}

Lo scopo della ricerca era di confrontare l'efficacia di modelli previsionali del default della PI sviluppati utilizzando congiuntamente ratios economico-finanziari e variabili inerenti il "territorio di insediamento" e/o la "relazione impresa-territorio" (modelli 2, 3 e 4) con quella di un modello costruito impiegando esclusivamente ratios economico-finanziari (modello 1 ).

11 Il principale vantaggio del modello Logit rispetto alla analisi discriminante multivariata risiede nella minore rigidità delle ipotesi sottostanti: l'unica condizione richiesta è infatti l'indipendenza delle variabili esplicative, mentre non risultano necessarie né la normalità della distribuzione delle variabili indipendenti, né l'uniformità delle matrici di varianza e covarianza nei gruppi. 
In prima battuta i modelli sono stati elaborati applicando la regressione logistica al training sample aggregato. Successivamente abbiamo suddiviso il training sample in 4 sotto-campioni (delimitati in funzione della dimensione d'impresa) ed abbiamo provato ad applicare la regressione in modo separato a ciascuno di tali sottocampioni. Per tutti i modelli elaborati ai diversi livelli di aggregazione i coefficienti sono risultati significativi all'1, 5 o 10 per cento e i segni osservati sono risultati coerenti con quelli attesi.

Tab. 6: Test sull'holdout sample dei modelli previsionali elaborati sul training sample aggregato (valori percentuali)

\begin{tabular}{|c|c|c|c|c|c|c|}
\hline \multirow[b]{2}{*}{ Modello } & \multirow{2}{*}{\multicolumn{2}{|c|}{ Stato osservato }} & \multicolumn{2}{|c|}{ Stato predetto } & \multirow{2}{*}{$\begin{array}{c}\text { Imprese } \\
\text { correttamente } \\
\text { classificate }\end{array}$} & \multirow{2}{*}{$\begin{array}{l}\text { Incremento di } \\
\text { accuratezza } \\
\text { previsionale su } \\
\text { Modello } 1\end{array}$} \\
\hline & & & 1 & 0 & & \\
\hline \multirow{2}{*}{ Modello 1} & Imprese in default & 1 & 75,5 & 24,5 & \multirow[b]{2}{*}{65,0} & \\
\hline & Imprese non in default & 0 & 43,9 & 56,1 & & \\
\hline \multirow{2}{*}{ Modello 2} & Imprese in default & 1 & 76,3 & 23,7 & \multirow{2}{*}{67,2} & \multirow{2}{*}{3,3} \\
\hline & Imprese non in default & 0 & 40,6 & 59,4 & & \\
\hline \multirow{2}{*}{ Modello 3} & Imprese in default & 1 & 78,9 & 21,1 & \multirow{2}{*}{71,5} & \multirow{2}{*}{10,1} \\
\hline & Imprese non in default & 0 & 34,7 & 65,3 & & \\
\hline \multirow{2}{*}{ Modello 4} & Imprese in default & 1 & 79,9 & 20,1 & \multirow{2}{*}{74,4} & \multirow{2}{*}{14,5} \\
\hline & Imprese non in default & 0 & 30,2 & 69,8 & & \\
\hline
\end{tabular}

Fonte: ns. elaborazione

La Tabella 6 mostra il grado di accuratezza previsionale fatto rilevare dai modelli previsionali elaborati applicando la regressione logistica sul training sample aggregato. In linea con l'ipotesi 1A, il modello elaborato utilizzando congiuntamente i ratios economico-finanziari e le variabili inerenti il "territorio di insediamento" (modello 2) consente di ottenere, rispetto al modello elaborato impiegando i soli ratios economico-finanziari (Modello 1), un incremento del livello di accuratezza previsionale pari al 3,3\%, con una riduzione dell'errore di tipo I (la percentuale di imprese in default erroneamente classificate) del 3,3\% ed una riduzione dell'errore di Tipo II (percentuale di imprese non in default erroneamente classificate) pari al 7,5\%.

Il modello elaborato utilizzando congiuntamente i ratios economico-finanziari e le variabili inerenti la "relazione impresa-territorio" (modello 3) fa rilevare, rispetto al modello 1, un incremento del grado accuratezza previsionale pari al 10,1\%, una riduzione dell'errore di tipo I del $13,9 \%$ ed una riduzione dell'errore di Tipo II del $21,0 \%$. Risulta quindi confermata anche l'ipotesi $1 \mathrm{~B}$.

Anche l'ipotesi di ricerca 1C risulta confermata: il modello 4 (quello costruito utilizzando congiuntamente ratios economico-finanziari, variabili inerenti il "territorio di insediamento" e variabili inerenti la "relazione impresa-territorio") consente infatti di ottenere i più elevati livelli di accuratezza previsionale: rispetto al modello 1 l'efficacia complessiva cresce del 14,5\% e gli errori di Tipo I e di Tipo II si riducono rispettivamente del $18 \%$ e del $31 \%$. Gli elevati valori assunti dall'errore di tipo II (da un minimo del 30,2\% per il modello 4 ad un massimo del 43,9\% per il modello 1) sono probabilmente dovuti al criterio restrittivo da noi adottato ai fini 
della definizione dell'evento di default: l'ingresso in procedura concorsuale giunge spesso dopo che si è manifestato lo stato di crisi irreversibile che già identifica uno stato di insolvenza di fatto.

Quando sono elaborati separatamente per ciascuna classe dimensionale tutti i modelli evidenziano una capacità previsionale significativamente più elevata rispetto a quella mostrata dalle funzione decisionali elaborate su base aggregata (Tabella 7), risultando così confermata anche l'ipotesi di ricerca n. 2 .

Tab. 7: Test sull'holdout sample dei modelli previsionali elaborati per classe dimensionale (valori percentuali)

\begin{tabular}{|c|c|c|c|c|c|c|}
\hline & & $\begin{array}{c}\text { Dimensione } \\
1\end{array}$ & $\begin{array}{c}\text { Dimensione } \\
2\end{array}$ & $\begin{array}{c}\text { Dimensione } \\
3 \\
\end{array}$ & $\begin{array}{c}\text { Dimensione } \\
4 \\
\end{array}$ & Totale \\
\hline \multirow{3}{*}{$\begin{array}{l}\text { Modello } \\
\quad 1\end{array}$} & Imprese correttamente classificate & 68,2 & 71,2 & 74,6 & 77,0 & 71,5 \\
\hline & $\begin{array}{l}\text { Imprese non in default } \\
\text { correttamente classificate }\end{array}$ & 60,1 & 62,5 & 63,4 & 67,1 & 62,3 \\
\hline & $\begin{array}{l}\text { Imprese in default correttamente } \\
\text { classificate }\end{array}$ & 78,7 & 82,6 & 85,5 & 87,7 & 82,3 \\
\hline \multirow{4}{*}{$\begin{array}{l}\text { Modello } \\
\quad 2\end{array}$} & Imprese correttamente classificate & 71,5 & 72,7 & 74,7 & 77,1 & 73,2 \\
\hline & $\begin{array}{l}\text { Incremento di accuratezza } \\
\text { previsionale su Modello } 1\end{array}$ & 4,8 & 2,1 & 0,1 & 0,1 & 2,4 \\
\hline & $\begin{array}{l}\text { Imprese non in default } \\
\text { correttamente classificate }\end{array}$ & 64,2 & 65,6 & 65,9 & 67,7 & 65,3 \\
\hline & $\begin{array}{l}\text { Imprese in default correttamente } \\
\text { classificate }\end{array}$ & 78,8 & 82,7 & 85,6 & 88,0 & 82,4 \\
\hline \multirow{4}{*}{$\begin{array}{l}\text { Modello } \\
3\end{array}$} & Imprese correttamente classificate & 77,2 & 77,3 & 77,2 & 77,3 & 77,3 \\
\hline & $\begin{array}{l}\text { Incremento di accuratezza } \\
\text { previsionale su Modello } 1\end{array}$ & 13,2 & 8,6 & 3,5 & 0,5 & 8,1 \\
\hline & $\begin{array}{l}\text { Imprese non in default } \\
\text { correttamente classificate }\end{array}$ & 70,5 & 71,6 & 72,0 & 71,0 & 71,2 \\
\hline & $\begin{array}{l}\text { Imprese in default correttamente } \\
\text { classificate }\end{array}$ & 82,4 & 84,9 & 85,7 & 88,1 & 84,4 \\
\hline \multirow{4}{*}{$\begin{array}{l}\text { Modello } \\
\quad 4\end{array}$} & Imprese correttamente classificate & 80,6 & 79,0 & 77,5 & 77,4 & 79,1 \\
\hline & $\begin{array}{l}\text { Incremento di accuratezza } \\
\text { previsionale su Modello } 1\end{array}$ & 18,1 & 11,0 & 3,8 & 0,6 & 10,7 \\
\hline & $\begin{array}{l}\text { Imprese non in default } \\
\text { correttamente classificate }\end{array}$ & 74,6 & 74,0 & 73,9 & 72,0 & 74,0 \\
\hline & $\begin{array}{l}\text { Imprese in default correttamente } \\
\text { classificate }\end{array}$ & 83,9 & 84,9 & 85,8 & 88,3 & 85,1 \\
\hline
\end{tabular}

Fonte: ns. elaborazione

Il modello 1 fa rilevare tassi globali di accuratezza previsionale crescenti al crescere delle dimensioni aziendali (dal $68 \%$ per le imprese della classe dimensionale minore al $77 \%$ per le imprese appartenenti alla classe dimensionale 4) ed un tasso globale di corretta classificazione pari al 71,5\%, con un incremento del grado di accuratezza previsionale pari al $10 \%$ ed una riduzione dell'errore di Tipo I particolarmente marcata $(27,8 \%)$ rispetto ai risultati fatti rilevare dalla funzione logistica elaborata su base aggregata.

Il modello 2 fa rilevare un tasso globale di corretta classificazione pari al 73,2\% (con tassi anche in questo caso crescenti al crescere delle dimensioni aziendali). Rispetto ai risultati fatti rilevare dalla funzione decisionale elaborata sul training sample aggregato si rilevano un incremento del grado di accuratezza previsionale del 
9\%, una riduzione dell'errore di Tipo I del $26 \%$ ed una riduzione dell'errore di tipo II del 14\%. Rispetto al Modello 1:

a) il grado di accuratezza previsionale cresce per la classe dimensionale $2(+2,10 \%)$ e, soprattutto, per la classe dimensionale $1(+4,8 \%)$, mentre non subisce variazioni significative con riferimento alle classi dimensionali superiori;

b) l'errore di tipo II si riduce complessivamente dell' $8 \%$ (10\% per la classe dimensionale inferiore, $2 \%$ per quella superiore), mentre l'errore di tipo I rimane sostanzialmente invariato $(17,6 \%)$.

Il modello 3 fa rilevare un tasso globale di corretta classificazione pari al 77,3\%. Rispetto ai risultati della formula predittiva elaborata su base aggregata si rileva un incremento del grado di accuratezza previsionale dell' $8 \%$, una riduzione dell'errore di Tipo I del $26 \%$ ed una riduzione dell'errore di tipo II del $17 \%$. Rispetto al Modello 1:

a) il miglioramento del livello di accuratezza previsionale è più elevato per le classi dimensionali inferiori $(+13 \%$ per la classe dimensionale $1,+9 \%$ per la Classe dimensionale $2,+3,5 \%$ per la classe $3,+0,5 \%$ per la classe 4 ), con il risultato che l'efficacia della formula previsionale risulta sostanzialmente la stessa nelle diverse classi $(77 \%)$;

b) oltre all'errore di tipo II (-24\%), si riduce sensibilmente anche l'errore di tipo I ($12 \%$ in totale; $-17 \%$ per la classe dimensionale inferiore).

Il modello 4 è quello che fa rilevare il tasso globale di corretta classificazione più elevato $(79,1 \%)$, con un incremento della efficacia previsionale di oltre il $6 \%$, una riduzione dell'errore di Tipo I del $26 \%$ ed una riduzione dell'errore di tipo II del $14 \%$ rispetto ai risultati della funzione logistica elaborata su base aggregata. Rispetto ai modelli 1 e 2 la relazione tra efficacia previsionale e classe dimensionale si inverte $(77 \%$ per la classe dimensionale superiore, $81 \%$ per la classe dimensionale inferiore). Rispetto al Modello 1 l'efficacia previsionale cresce in modo particolarmente accentuato per le classi dimensionali inferiori $(+18 \%$ per la classe dimensionale $1,+11 \%$ per la classe dimensionale 2) e gli errori di tipo I e di tipo II si riducono rispettivamente del $16 \%$ e del $31 \%$ (la prima classe dimensionale fa rilevare le riduzioni più marcate: $-24 \%$ per l'errore di tipo I e $-36 \%$ per l'errore di tipo II).

\section{Conclusioni}

È oggi particolarmente sentita l'esigenza di migliorare l'efficacia dei modelli utilizzati dalle agenzie di rating e dagli intermediari finanziari per stimare il merito creditizio di imprese, persone fisiche, prodotti finanziari e programmi di investimento (Banca Centrale Europea, 2012).

La nostra ricerca affronta il tema del contributo che l'utilizzo delle variabili qualitative (tangibili ed intangibili) inerenti il "territorio di insediamento" e la "relazione impresa-territorio" può fornire alla efficacia dei modelli previsionali del default della PI. I risultati ottenuti dimostrano che tale contributo è significativo e permette di compensare l'elevato grado di opacità che tipicamente caratterizza 
l'informazione contabile della PI. Rispetto al caso delle formule previsionali stimate utilizzando le sole variabili quantitative (indici di bilancio), il grado di accuratezza predittiva dei modelli: i) cresce in misura lieve quando si introduce l'utilizzo delle variabili qualitative relative al "territorio" $(+3 \%$ quando la funzione logistica è elaborata sul campione aggregato; $+2 \%$ quando la funzione logistica è elaborata per singola classe dimensionale); ii) cresce in misura molto più significativa (rispettivamente $+10 \%$ e $+8 \%$ ) quando si introduce l'utilizzo delle variabili relative alla "relazione impresa-territorio"; iii) cresce in misura ancor più rilevante (rispettivamente $+14 \%$ e $+11 \%$ ) quando si introduce l'utilizzo di entrambe le categorie di variabili qualitative. La stima di funzioni previsionali distinte per classe dimensionale si associa, in generale, ad un miglioramento del grado di efficacia dei modelli (Tabella 8). Mentre per il modello 1 l'accuratezza previsionale cresce al crescere della dimensione d'impresa, per il modello 4 tale tendenza risulta invertita: il contributo delle variabili relative alla relazione impresa-territorio risulta quindi tanto più elevato quanto minore è la dimensione d'impresa.

Tab. 8: Test sull'holdout sample dei modelli previsionali elaborati ai due livelli di aggregazione: tassi di corretta classificazione (valori percentuali)

\begin{tabular}{|ccccc|}
\hline Livello di analisi & Modello 1 & Modello 2 & Modello 3 & Modello 4 \\
\hline Campione Aggregato & 65,0 & 67,2 & 71,5 & 74,4 \\
\hline Classe Dimensionale & 71,5 & 73,2 & 77,3 & 79,1 \\
\hline
\end{tabular}

Fonte: ns. elaborazione

Dal punto di vista delle implicazioni manageriali il lavoro evidenzia l'opportunità, per gli istituti finanziari, di integrare le variabili qualitative relative al territorio e, soprattutto, quelle relative alla relazione impresa-territorio nei processi di elaborazione dei propri modelli di credit rating. L'impiego congiunto di tali categorie di variabili consente di ottenere una marcata riduzione dell'errore di "Tipo II" (percentuale di imprese per le quali il modello prevede un default che poi non ha effettivamente luogo) e può quindi aiutare a limitare la tendenza ad assumere comportamenti di eccessivo razionamento del credito. I risultati della ricerca evidenziano inoltre l'esigenza di tarare la funzione decisionale sulla base di campioni di imprese sufficientemente omogenei dal punto di vista della dimensione aziendale: il pooling di classi dimensionali impoverisce la capacità predittiva del modello e questo vale, a fortiori, nel caso di modelli che l'intermediario finanziario acquisisce da soggetti esterni e/o costruisce su campioni non rappresentativi dell'universo con il quale si trova normalmente ad operare. Dall'esperimento qui condotto in cross section si può altresì desumere (per via analogica) l'opportunità di aggiornare con frequenza la stima della funzione di decisione.

La presente ricerca manifesta alcuni limiti: i dati relativi alle variabili qualitative sono influenzati dalle percezioni soggettive del management delle imprese oggetto di analisi; non sono state impiegate variabili qualitative ulteriori quali quelle inerenti le strategie competitive adottate, le competenze del management, gli attributi interorganizzativi; non sono state utilizzate variabili quantitative relative all'andamento della relazione impresa-sistema finanziario. 


\section{Bibliografia}

ACS Z.J., ANSELIN L., VARGA A. (2002), "Patents and Innovation Counts as Measures of Regional Production of New Knowledge", Research Policy, n. 31, pp. 1069-1085.

AHUJA G. (2000), "Collaboration Networks, Structural Holes, and Innovation: a Longitudinal Study", Administrative Science Quarterly, vol. 45, n. 3, pp. 425-455.

ALESSANDRINI P., PRESBITERO A.F., ZAZZARO A. (2006), Banks, Distances and Financing Constraints for Firms, Università Politecnica delle Marche - Quaderni di Discussione.

ALESSANDRINI P., PRESBITERO A.F., ZAZZARO A. (2007), Bank Size or Distance: What Hampers Innovation Adoption by SMEs?, Università Politecnica delle Marche Quaderni di Discussione.

ALTMAN E.I. (1968), "Financial ratios, discriminant analysis and the prediction of corporate bankruptcy", The Journal of Finance, vol. 23, n. 4, pp. 589-609.

ALTMAN E.I. (1993), Corporate Financial Distress and Bankruptcy $\left(2^{\text {nd }}\right.$ ed.), Wiley, New York, NY.

ALTMAN E.I., HALDEMAN R., NARAYANAN P. (1977), “ZETA Analysis: A New Model to Identify Bankruptcy Risk of Corporations", Journal of Banking and Finance, vol. 1, n. 1, pp. 29-54.

ALTMAN E.I., SABATO G. (2007), "Modeling Credit Risk for SMEs: Evidence from the US Market", Abacus, vol. 43, n. 2, pp. 332-357.

ALTMAN E.I., SABATO G., WILSON N. (2010), "The Value of Non-Financial Information in Small and Medium-Sized Enterprise Risk Management", The Journal of Credit Risk, vol. 6, n. 2, pp. 95-127.

ALTMAN E., SAUNDERS A. (1996), "Credit Risk Measurement: Development over the Last 20 Years", Working Paper Series, New York.

ASHEIM B., COENEN L. (2005), "Knowledge Bases and Regional Innovation Systems: Comparing Nordic Clusters", Research Policy, vol. 34, n. 8, pp. 1173-1190.

ASHEIM B., ISAKSEN A. (2002), "Regional Innovation Systems: The Integration of Local 'Sticky' and Global 'Ubiquitous' Knowledge”, The Journal of Technology Transfer, vol. 27, n. 1, pp. 77-86.

ASHEIM B.T., GERTLER M.S. (2005), “The Geography of Innovation: Regional Innovation Systems", in Fagerberg J., Mowery D., Nelson R., (eds.), The Oxford Handbook of Innovation, Oxford University Press, Oxford.

AUTIO E. (1998), "Evaluation of RTD in Regional Systems of Innovation", European Planning Studies, vol. 6, n. 2, pp. 131-140.

BARILE S. (2009), Management sistemico vitale, Giappichelli, Torino, II ed.

BACCARANI C., GOLINELLI G.M. (1993), Tratti del divenire dei distretti industriali, Istituto Tagliacarne, Roma.

BACCARANI C., GOLINELLI G.M. (2011), "Per una rivisitazione delle relazioni tra impresa e territorio", Sinergie, n. 84, pp. VII-XIII.

BACK B., LAITINEN T., SERE K. (1996), "Neural Networks and Genetic Algorithms for Bankruptcy Predictions", Expert System With Applications, vol. 11, n. 4, pp. 407-413.

BAFFIGI A., PAGNINI M., QUINTILIANI F. (2000), "Localismo bancario e distretti industriali: assetto dei mercati del credito e finanziamento degli investimenti", in Signorini F.L. (a cura di), Lo sviluppo locale, Donzelli, Roma.

BARNES P. (1982), "Methodological Implications of Non-Normality Distributed Financial Ratios", Journal of Business Finance and Accounting, vol. 9, n. 1, pp. 51-62. 
BANCA CENTRALE EUROPEA (2012), Parere del 2 aprile 2012 in merito alla proposta di regolamento che modifica il regolamento (CE) n. 1060/2009 relativo alle agenzie di rating del credito, Gazzetta ufficiale dell'Unione europea C167/2 del 13 giugno 2012.

BECATTINI G. (1979), "Dal settore industriale al distretto industriale. Alcune considerazioni sull'unità di indagine dell'economia industriale", Rivista di Economia e Politica Industriale, vol. 5, n. 1, pp. 35-48.

BECATTINI G. (1989), Modelli locali di sviluppo, Il Mulino, Bologna.

BECATTINI G. (1990), "The Marshallian Industrial District as a Socio-Economic Notion", in Pyke F., Becattini G., Sengenberger W., (a cura di), Industrial Districts and Inter-firm Co-operation in Italy, ILO, Ginevra.

BECATTINI G. (2000), Il distretto industriale. Un nuovo modo di interpretare il cambiamento economico, Rosenberg \& Sellier, Torino.

BECATTINI G., RULLANI E. (1993), "Sistema locale e mercato globale", Economia $e$ politica industriale, n. 80 , pp. 25-48.

BEHR P., GÜTTLER A. (2007), "Credit Risk Assessment and Relationship Lending: An Empirical Analysis of German Small and Medium-Sized Enterprises", Journal of Small Business Management, vol. 45, n. 2, pp. 194-213.

BELLANDI M. (2009), "External Economies, Specific Public Goods and Policies", in Becattini G., Bellandi M., De Propris L., (eds), A handbook of industrial districts, Edward Elgar, Cheltenham.

BELUSSI F., GOTTARDI G., RULLANI E. (2003), The Technological Evolution of Industrial Districts, Kluwer, Boston.

BELUSSI F., PILOTTI L. (2008), "Creazione, produzione, trasferimento di conoscenze nei SPL tra tacitness e codified knowledge in una prospettiva ecologica del valore", in Centazzo R., Pasini F., (a cura di), I sistemi produttivi locali: evidenze empiriche e politiche di sviluppo, Franco Angeli, Milano.

BLUM M. (1974), "Failing Company Discriminant Analysis", Journal of Accounting Research, vol. 12, n. 1, pp. 1-25.

BOARI C., LIPPARINI A. (1999), "Network within Industrial Districts: Organising Knowledge Creation and Transfer by Means of Moderate Hierarchies", Journal of Management and Governance, n. 3, pp. 339-360.

BORTOLUZZI G., TRACOGNA A. (2011), "Processi di innovazione e fattori di competitività nei cluster internazionali della nautica", Sinergie, n. 84, pp. 89-118.

BOSCHMA R. (2005), "Proximity and Innovation: A Critical Assessment", Regional Studies, vol. 39 , n. 1, pp. 61-74

BOTTINELli L., PAVIONE E. (2010), Distretti industriali e cluster tecnologici: strategie emergenti di valorizzazione della ricerca e dell'innovazione, Giuffrè, Milano.

BOYD B.K. (1995), "CEO Duality and Firm Performance: A Contingency Model”, Strategic Management Journal, vol. 16, n. 4, pp. 301-312.

BOZEMAN B. (2000), "Technology Transfer and Public Policy: A Review of Research and Theory", Research Policy, n. 29, pp. 627-655.

BRACZYK H.J., COOKE M.H. (1998), Regional Innovation Systems: The Role of Governances in a Globalized World, Routledge, New York.

BRADY K.O. (2002), "Corporate Reputation and Sustainability: Creating Value by Unifying Two Disciplines", Ethical Corporation Magazine, n. 9, pp. 26-27.

BRESCHI S., MALERBA F. (2005), Clusters, Networks and Innovation, Oxford University Press, Oxford.

BRUSCO S. (1982), "The Emilian Model: productive Decentralisation and Social Integration", Cambridge Journal of Economics, vol. 6, n. 2, pp. 167-184. 
BRUSCO S. (1989), Piccole imprese e distretti industriali, Rosemberg \& Sellier, Torino.

BRUSCO S. (1999), "The Rules of the Game in Industrial Districts", in Grandori A., (ed), Interfirm Networks: Organization and Industrial Competitiveness, Routledge, London.

BUESA M., HEIJS J., BAUMERT T. (2010), “The Determinants of Regional Innovation in Europe: A Combined Factorial and Regression Knowledge Production Function Approach", Research Policy, vol. 39, n. 6, pp. 722-735.

CAIROLI M.G. (2011), Il marketing territoriale. Strategie per la competitività sostenibile del territorio, Franco Angeli, Milano.

CAMAGNI R. (1991), Innovation Networks: Spatial Perspectives, Belhaven-Pinter, London.

CAMUFFO A., GRANDINETTI R. (2006), "I distretti industriali come sistemi locali di innovazione", Sinergie, n. 69, pp. 33-60.

CAPELLO R. (2002), "Spatial and Sectorial Characteristics of Relational Capital in Innovation Activity”, European Planning Studies, n. 10, pp. 177-200.

CARTER R., VAN AUKEN H. (2006), "Small Firm Bankruptcy", Journal of Small Business Management, vol. 44, n. 4, pp. 493-512.

CHAGANTI R.S., MAHAJAN V., SHARMA S. (1985), "Corporate Board Size, Composition and Corporate Failures in Retailing Industry", Journal of Management Studies, n. 22, pp. 400-416.

CHIAVERSIO M., MICELLI S. (2007), "Oltre il distretto come sistema: le strategie delle imprese fra locale e globale", in Guelpa F., Miceli S., I distretti industriali del terzo millennio, Il Mulino, Bologna.

CIAMPI F. (1994), "Squilibri di assetto finanziario nelle P.M.I. Finanziamenti e contributi della Comunità Europea", Studi e Informazioni, Supplemento al n. 3, pp. 1-202.

CIAMPI F., GORDINI N. (2008), "Using Economic-Financial Ratios for Small Enterprise Default Prediction Modeling: An Empirical Analysis", 2008 Oxford Business \& Economics Conference Proceedings, Association for Business and Economics Research (ABER), pp. 1-21.

CIAMPI F., GORDINI N. (2009), "Default Prediction Modeling for Small Enterprises: Evidence from Small Manufacturing Firms in Northern and Central Italy", Oxford Journal, vol. 8, n. 1, pp. 13-29.

CIAMPI F., GORDINI N. (2013), Small Enterprise Default Prediction Modeling through Artificial Neural Networks: An Empirical Analysis of Italian Small Enterprises, Journal of Small Business Management, vol. 51, n.1, pp. 23-45.

COLEMAN J.S. (1988), "Social Capital in the Creation of Human Capital", American Journal of Sociology, n. 94, pp. 95-120.

COLEMAN J.S. (1990), Foundations of Social Theory, Belknap Press of Harvard University Press, Cambridge, MA.

COOKE P. (2001), "Regional Innovation Systems, Clusters and the Knowledge Economy", Industrial and Corporate Change, vol. 10, n. 4, pp. 945-973.

COOKE P. (2002), Knowledge Economies. Clusters, Learning and Cooperative Advantage, Routledge, London.

COOKE P., MORGAN K. (1998), The Associational Economy: Firms, Regions and Innovation, Oxford University Press, Oxford.

COOKE P., URANGA M.G., ETXEBARRIA G. (1997), "Regional Innovation Systems: Institutional and Organizational Dimensions", Research Policy, n. 26, pp. 475-491.

COOPER A., GASCON J., WOO C. (1991), “A Resource-Based Prediction of New Venture Survival and Growth", Proceedings Academy of Management, Summer, pp. 113-119. 
CORÒ G., MICELLI S. (2006), I nuovi distretti produttivi. Innovazione, internazionalizzazione e competitività dei territori, Marsilio, Venezia.

CORÒ G., MICELLI S. (2007), "I distretti industriali come sistemi locali dell'innovazione: imprese leader e nuovi vantaggi competitive dell'industria italiana", Economia Italiana, n. 1, pp. 321-356.

CORÒ G., RULLANI E. (1998), Percorsi locali di internazionalizzazione. Competenze e auto-organizzazione dei distretti industriali del Nord-Est, Franco Angeli, Milano.

CREVOISIER O., CAMAGNI R. (2000), Les Milieu Urbains: Innovation, Systèmes de Production et Anchorage, EDES, Neuchâtel, Switzerland.

CROUHY M., GALAI D., MARK R. (2001), "Prototype Risk Rating System”, Journal of Banking and Finance, vol. 25, n. 1, pp. 47-95.

DAGNINO G.B., D’ALLURA G.M., FARACI R., PISANO V. (2011), "La localizzazione dei processi innovativi ad alto contenuto tecnologico: il ruolo delle imprese "àncora" fra sistemi locali e network globali", Sinergie, n. 84, pp. 179-207.

DAILY C., DALTON R. (1994), "Bankruptcy and Corporate Governance: The Impact of Board Composition and Structure", Academy of Management Journal, vol. 37, n. 6, pp. 1603-1617.

DE MAIO A., MAGGIORE E. (1992), Organizzare per innovare, Etas Libri, Milano.

DEAKIN E.B. (1972), “A Discriminant Analysis of Predictors of Business Failure”, Journal of Accounting Research, vol. 10, n. 1, pp. 167-179.

DEI OTTATI G. (1994), "Trust, Inter-Linking Transactions and Credit in the Industrial District", Cambridge Journal of Economics, vol. 18, n. 6, pp. 529-546.

DEI OTTATI G. (2003), "Fiducia e cambiamento economico nei distretti industriali. Il caso del distretto di Prato", Sviluppo Locale, vol. X, n. 23-24, pp. 82-105.

DENICOLAI S., CIOCCARELlI G., ZUCCHELLA A. (2010), "Reputation, Trust and Relational Centrality in Local Networks: An Evolutionary Geography Perspective", in Boschma R., Martin R., (eds), Handbook on evolutionary economic geography, Edward Elgar, UK.

DOLOREUX D., PARTO S. (2005), "Regional Innovation Systems: Current Discourse and Unresolved Issues", Technology in Society, vol. 27, n. 2, pp. 133-153.

DOSI G., FREEMAN C., NELSON R., SILVERBERG G., SOETE L. (1988), Technical Change and Economic Theory, LEM Book Series, Pisa.

DUNCAN R. (1976), "The Ambidextrous Organization: Designing Dual Structures for Innovation", The management of organization design: Strategies and implementation, vol. 1, pp. 167-188.

DYER J., SINGH H. (1998), "The Relational View: Cooperative Strategy and Sources of Interorganizational Competitive Advantage", Academy of Management Journal, vol. 23, n. 4, pp. 660-679.

EDMISTER R. (1972), “An Empirical Test of Financial Ratio Analysis for Small Business Failure Prediction", Journal of Financial and Quantitative Analysis, vol. 7, n. 2, pp. 1477-1493.

ELY J., VALIMAKI J. (2002), "Bad Reputation", NAJ Economics, vol. 4, n. 5, pp. 1-24.

ENGELMANN D., FISCHBACHER U. (2003), "Indirect Reciprocity and Strategic Building in an Experimental Helping Game", Working Paper, Egham.

EVANS D.S., LEIGHTON L. (1989), "Some Empirical Aspects of Entrepreneurship", American Economic Review, vol. 79, n. 3, pp. 519-535.

FERGUSON T.D., DEEPHOUSE D.L., FERGUSON W.L. (2000), "Do Strategic Groups Differ in Reputation?”, Strategic Management Journal, vol. 21, n. 12, pp. 1195-1214. 
FERRI P., LUSIANI M., SANTONI S. (2009), "The Contingent Value of Bridging and Bonding Social Capital on Firm Performance: A Meta-Analysis", Academy of Management Meeting, Chicago, Illinois.

FOSS N.J. (1996), "Higher-Order Industrial Capabilities and Competitive Advantage", Journal of Industry Studies, vol. 3, n. 1, pp. 1-20.

GALASKIEWICZ J. (1985), Social Organization of an Urban Grants Economy, Academic Press, Orlando, FL.

GARGIULO M., BENASSI M. (2000), "Trapped in Your Own Net? Network Cohesion Structural Holes, and the Adaptation of Social Capital", Organization Science, vol. 11, n. 2, pp. 183-196.

GERINGER J.M., TALLMAN S., OLSEN D.M. (2000), "Product and International Diversification among Japanese Multinational Firms", Strategic Management Journal, n. 21, pp. 51-80.

GHOSHAL S., BARLETT C. (1994), "Linking Organizational Context and Managerial Action: The Dimension of Quality of Management", Strategic Management Journal, vol. 15, Supplement S2, pp. 91-115.

GLAESER E., KALLAL H., SCHEINKMAN J., SCHLEIFER A. (1992), "Growth in Cities", Journal of Political Economy, vol. 100, n. 6, pp. 1126-1152.

GOLINELLI C.M. (2002), Il territorio sistema vitale. Verso un modello di analisi, Giappichelli, Torino.

GOLINELLI G.M. (2010), Viable System Approach, Governing Business Dynamics, Kluwer, Cedam, Padova.

GOLINELLI G.M. (2011), L'Approccio Sistemico Vitale (ASV) al governo dell'impresa, Cedam, Padova.

GOLINELlI G.M., PASTORE A., GATTI M., MASSARONI E., VAGNANI G. (2002), "The Firm as a Viable System: Managing Inter-Organisational Relationship, Sinergie, n. 58, pp. 65-98.

GRANDINETTI R., TABACCO R. (2003), "I distretti industriali come laboratori cognitivi", Sviluppo Locale, vol. 10, n. 22, pp. 33-60.

GRANOVETTER M. (1985), "Economic Action and Social Structure: The Problem of Embeddedness", American Journal of Sociology, vol. 91, n. 3, pp. 481-510.

GRECO S., MATARAZZO B., SLOWINSKI R. (1998), “A New Rough Set Approach to Evaluation of Bankruptcy Risk", in Zopounidis C., Operational Tools in the Management Of Financial Risks, Kluwer Academic Publisher, Dordrecht.

GULATI R. (1998), “Alliances and Networks”, Strategic Management Journal, vol. 19, n. 4 , pp. 293-317.

GULATI R., NOHRIA N., ZAHEER A. (2000), "Strategic Networks", Strategic Management Journal, vol. 21, n. 3, pp. 203-215.

HASWELL S., HOLMES S. (1989), "Estimating the Small Business Failure Rate: A Reappraisal", Journal of Small Business Management, vol. 27, n. 3, pp. 68-74.

HENDERSON V. (1996), Externalities and Industrial Development, Brown University, Mimeo.

HUNDLEY G., JACOBSON C. (1998), "The Effects of Keiretsu on Export Performance of Japanese Companies: Help or Hindrance?", Strategic Management Journal, vol. 19, n. 10, pp. 927-937.

JIMENEZ G., SALAS V., SAURINA J. (2007), Organizational Distance and Use of Collateral in Business Loans, Mimeo. 
JO H., HAN I., LEE H. (1997), “Bankruptcy Prediction Using Case-Based Reasoning, Neural Network and Discriminant Analysis”, Expert Systems With Applications, vol. 13, n. 2 , pp. 97-108.

KARELS G.V., PRAKASH A.J. (1987), "Multivariate Normality and Forecasting of Business Bankruptcy", Journal of Business Finance \& Accounting, vol. 14, n. 4, pp. 573-595.

KOH H.C. (1996), "Testing Hypotheses of Entrepreneurial Characteristics: A Study of Hong Kong MBA Students”, Journal of Managerial Psychology, vol. 11, n. 3, pp. 12-25.

KRUGMAN P. (1991a), "Increasing Returns and Economic Geography”, Journal of Political Economy, vol. 99, n. 3, pp. 483-499.

KRUGMAN P. (1991b), Geography and Trade, MIT Press, Cambridge, MA.

KRUGMAN P. (1995), Development, Geography and Economic Theory, MIT Press, Cambridge.

LARSON C.M., CLUTE R.C. (1979), "The Failure Syndrome”, American Journal of Small Business, vol. 4, n. 2, pp. 35-43.

LAVIE D. (2006), “The Competitive Advantage of Interconnected Firms: An Extension of the Resource-Based View", Academy of Management Review, vol. 31, n. 3, pp. 638658.

LAZERSON M.H., LORENZONI G. (1999), "The Firms that Feed Industrial Districts: A Return to the Italian Source", Industrial and Corporate Change, vol. 8, n. 2, pp. 235 266.

LEE K.C., HAN I., KWON Y. (1996), "Hybrid Neural Network Models for Bankruptcy Predictions", Decision Support Systems, vol. 18, n. 1, pp. 63-72.

LEIPONEN A., BYMA J. (2009), "If You Cannot Block, You Better Run: Small Firms, Cooperative Innovation, and Appropriation Strategies", Research Policy, vol. 38, n. 9 , pp. 1478-1488.

LIBERTI J. M., MIAN A. (2006), Estimating the Effect of Hierarchies on Information Use, Mimeo.

LIPPARINI A., LORENZONI G. (1996), "Le organizzazioni ad alta intensità relazionale. Riflessioni sui processi di "learning by interacting" nelle aree ad alta concentrazione di imprese”, L'Industria, vol. 17, n. 4, pp. 817-839.

LORENZ E. (1992), "Trust, Community, and Co-Operation. Toward a Theory of Industrial Districts", in Storper M., Scott A., (eds.), Pathways to Industrialization and Regional Development, Routledge, London.

LORENZONI G. (1997), "Le reti interimpresa come forma organizzativa”, in Lomi A. (a cura di), L'analisi relazionale delle organizzazioni. Riflessioni teoriche ed esperienze empiriche, Il Mulino, Bologna.

LUGARESI S., ROTONDI Z. (2007), “Internazionalizzazione e finanziamento dei distretti industriali", Economia Italiana, n. 1, pp. 105-125.

LUNDVALL B.A. (1992), National Systems of Innovation. Towards a Theory of Innovation and Interactive Learning, Pinter Publishers, London.

LUSSIER R. (1995), “A Non Financial Business Success versus Failure Prediction Model for Young Firms", Journal of Small Business Management, vol. 33, n. 1, pp. 8-20.

MAGGIONI M., UBERTI T.E. (2005), "International Networks of Knowledge Flows: An Econometric Analysis", Papers in Economics and Evolution, Max Planck Institute, Jena.

MAILLAT D. (1995), "Territorial Dynamics, Innovative Milieu and Regional Policy", Entrepreneurship and Regional Development, vol. 7, n. 2, pp. 157-165.

MAILLAT D., PERRIN J.C. (1992), Entreprises Innovatrices et Développement Territorial, GREMI EDS, Neuchâtel, Switzerland. 
MAILLAT D., QUEVIT M., SENN L. (1993), Réseaux D'innovation e Milieux Innovateurs: Un Pari Pour le Développement Régional, GREMI EDES, Neuchâtel, Switzerland.

MALECKI E. (1995), "Culture as Mediator of Global and Local Forces", in Van Der Knaap B., Le Heron R., (eds), Human Resources and Industrial Spaces: A Perspective on Globalization and Location, John Wiley \& Sons, Chichester.

MALECKI E. (2000), "Soft Variables in Regional Science", Review of Regional Studies, vol. 30, n. 1, pp. 61-69.

MARSHALL A. (1919), Industry and Trade, MacMillan, London.

MARSHALL A. (1925), Principles of Economics 8th edn., MacMillan, London.

MASKELL P., MALMBERG A. (2007), "Myopia, Knowledge Development and Cluster Evolution", Journal of Economic Geography, vol. 7, n. 5, pp. 603-618.

MASKELL P., MALMBERG A. (1999), "Localised Learning and Industrial Competitiveness", Cambridge Journal of Economics, vol. 23, n. 2, pp. 167-185.

MCEVILY B., ZAHEER A. (1999), "Bridging Ties: A Source of Firm Heterogeneity in Competitive Capabilities", Strategic Management Journal, vol. 20, n. 12, pp. 11331156.

MELE R. (2011), “Enti locali e valorizzazione dell'imprenditorialità innovative", Sinergie, n. 84, pp. 119-122.

MICELLI S., CHIARVESIO M. (2003), "Processi di internazionalizzazione e strategie delle imprese distrettuali tra delocalizzazione e innovazione", Conferenza ICE: Internazionalizzazione e frammentazione della produzione dei distretti industriali, Roma.

MOLINA-MORALES F.X., MARTINEZ-FERNANDEZ M.T. (2003), "The Impact of Industrial District Affiliation on Firm Value Creation", European Planning Studies, vol. 11, n. 2, pp. 155-170.

MORAN P., GHOSHAL S. (1996), "Value Creation by Firms", Academy of Management Best Paper Proceedings, vol. 5, n. 1, pp. 41-45.

MUSTILLI M., CAMPANELLA F., SORRENTINO F. (2011), "La valutazione delle performance innovative dei sistemi locali di innovazione: il caso dell'aerospazio e dei nuovi materiali in Campania", Sinergie, n. 84, pp. 209-229.

NAHAPIET J., GHOSHAL S. (1998), "Social Capital, Intellectual Capital, and the Organizational Advantage", The Academy of Management Review, vol. 23, n. 2, pp. 242-266.

NOOTEBOOM B. (2002), Trust: Forms, Foundations, Functions, Failures and Figures, Edward Elgar, Cheltenham, UK and Northampton, MA, USA.

NOOTEBOOM B. (2004), Interfirm Collaboration, Learning and Networks, Routledge, London.

OHLSON J.A. (1980), "Financial Ratios and the Probabilistic Prediction of Bankruptcy", Journal of Accounting Research, vol. 18, n. 1, pp. 109-131.

OLMEDA I., FERNANDEZ E. (1997), "Hybrid Classifiers for Financial Multicriteria Decision Making: The Case of Bankruptcy Prediction", Computational Economics, vol. 10, n. 4 , pp. 317-335.

PAGNINI M. (2000), "I vincoli finanziari per le imprese distrettuali”, in Signorini F.L., (a cura di), Lo sviluppo locale, Donzelli, Roma.

PIORE M., SABEL C. (1984), The Second Industrial Divide: Possibilities for Prosperity, Basic books, New York.

POMPE P.P.M., BILDERBEEK J. (2005), "The Prediction of Bankruptcy of Small-And Medium-Sized Industrial Firms", Journal of Business Venturing, vol. 20, n. 6, pp. 847-868. 
PORTER M. (1990), The Competitive Advantage of the Nations, The Free Press, New York.

PORTER M.E. (1998), "Clusters and the New Economics in Competition", Harvard Business Review, vol. 76, n. 6, pp. 77-90.

PUTNAM R. (1993), "The Prosperous Community: Social Capital and Public Life", American Prospect, vol. 4, n. 13, pp. 35-42.

PUTNAM R. (1995), "Bowling Alone: America's Declining Social Capital", Journal of Democracy, vol. 6, n. 1, pp. 65-78.

QUADRIO CURZIO A., FORTIS M. (2002), Complessità e distretti industriali. Dinamiche, modelli, casi reali, Il Mulino, Bologna.

RAISCH S., BIRKINSHAW J., PROBST G., TUSHMAN M.L. (2009), “Organizational Ambidexterity: Balancing Exploitation and Exploration for Sustained Performance", Organization Science, vol. 20, n. 4, pp. 685-695.

ROBERTSON P.L., JACOBSON D., LANGLOIS R.N. (2009), "Innovation Processes and Industrial Districts”, in Becattini G., Bellandi M., De Propris L., (eds), A Handbook of Industrial Districts, Edward Elgar, Cheltenham.

ROLFO S. (1996), "Innovazione tecnologica e competitività internazionale: quale futuro per i distretti e le economie locali?", Working Paper Ceris-CNR.

ROTONDI Z. (2005), "Banche, finanziamento dello sviluppo e dell'innovazione e internazionalizzazione", in Bracchi G., Masciandaro D. (a cura di), Decimo Rapporto SFI. Le banche Italiane e la finanza per lo sviluppo: territori, imprese e famiglie, Edibank-Bancaria Editrice.

RULLANI E. (1996), "Reti globali e reti metropolitane per l'economia del Nordest", in Banco Ambrosiano Veneto (a cura di), Nord-Est: fattori di competitività, Il Mulino, Bologna.

RULLANI E. (2003), "I distretti industriali al tempo dell'economia globale”, Impresa e Stato, n. 63-64, pp. 80-83.

RULLANI E. (2006), “L’internazionalizzazione invisibile. La nuova geografia dei distretti e delle filiere produttive", Sinergie, n. 69, pp. 3-32.

RUSSO P.F., ROSSI P. (1999), "Costo e disponibilità del credito per le imprese nei distretti industriali”, Banca d'Italia - Temi di Discussione, n. 360, pp. 1-53.

SAKO M. (1991), "The Role of Trust in Japanese Buyer-Supplier Relationships", Ricerche Economiche, vol. XLV, n. 2-3, pp. 449-474.

SALCHENBERGE, L.M., CINAR E.M., LASH N.A. (1992), "Neural Networks: A New Tool for Predicting Thrift Failures", Decision Sciences, vol. 23, n. 4, pp. 899-916.

SANTONI S., ZANNI L. (2011), "Comportamenti di ricerca e innovazione nei distretti industriali: gli attori del cambiamento e l'accesso a "conoscenze distanti", Sinergie, n. 84, pp. 231-256.

SAURINA J., TRUCHARTE C. (2004), "The Impact of Basel II on Lending to Small-andMedium-Sized Firms: A Regulatory Policy Assessment Based on Spanish Credit Register Data", Journal of Financial Services Research, vol. 26, n. 2, pp. 121-144.

SAXENIAN A.L. (1994), Regional Advantage: Culture and Competition in Silicon Valley and Route 128, Harvard University Press, Cambridge, MA.

SCHILLACI C.E., GATTI M. (2011), “E pluribus unum: intenzionalità collettiva e governo dei sistemi territoriali", Sinergie, n. 84, pp. 21-45.

SCHILLACI C.E., ROMANO M., LONGO C. (2009), Hybrid Organizational Forms and Academic Entrepreneurship. The Evolution of Italian University Incubators, Giappichelli, Torino.

SIGNORINI F.L. (2000), Lo sviluppo locale, Donzelli, Roma. 
SMITKA M. (1991), Competitive Ties: Subcontracting in the Japanese Automotive Industry, Columbia University Press, New York.

SOBRERO M., ROBERTS E.B. (2001), "The Trade-Off between Efficiency and Learning in Interorganizational Relationships for Product Development", Management Science, vol. 47, n. 4, pp. 493-511.

SUCHMAN M. (1994), On Advice of Counsel: Law Firms and Venture Capital Funds as Information Intermediaries in the Structuration of Silicon Valley, Doctoral Dissertation, Stanford University.

TODTLING F., TRIPPL M. (2005), “One Size Fits All? Towards a Differentiated Regional Innovation Policy Approach”, Research Policy, vol. 34, n. 8, pp. 1203-1219.

TSAI W., GHOSHAL S. (1998), "Social Capital and Value Creation: The Role of Intrafirm Networks", Academy of Management Journal, vol. 41, n. 4, pp. 464-476.

UGHETTO E. (2006), "Territorial Proximity and Credit Constraints to Innovation: Evidence from Italian Firms", in Bracchi G., Masciandaro D., (a cura di), Undicesimo Rapporto SFI. Banche e geografia: nuove mappe produttive e metamorfosi del credito, Edibank-Bancaria Editrice, Milano.

UZZI B. (1996), "The Sources and Consequences of Embeddedness for Economic Performance of Organizations: The Network Effect”, American Sociological Review, vol. 1, n. 4, pp. 674-698.

UZZI B. (1997), "Social Structure and Competition in Interfirm Networks: The Paradox of Embeddedness", Administrative Science Quarterly, vol. 42, n. 2, pp. 339-365.

VALLINI C., CIAMPI F., GORDINI N. (2009), "Using Artificial Neural Networks Analysis for Small Enterprise Default Prediction Modeling: Statistical Evidence from Italian Firms", 2009 Oxford Business \& Economics Conference Proceedings, Association for Business and Economics Research (ABER), pp. 1-26.

VALLINI C., CIAMPI F., GORDINI N., BENVENUTI M. (2008), “Can Credit Scoring Models Effectively Predict Small Enterprise Default? Statistical Evidence from Italian firms", Proceedings of the 8th Global Conference on Business \& Economics, Association for Business and Economics Research (ABER), pp. 1-23.

VALLINI C., CIAMPI F., GORDINI N., BENVENUTI M. (2009), "Are Credit Scoring Models Able to Predict Small Enterprise Default? Statistical Evidence from Italian Small Enterprises", International Journal of Business \& Economics, vol. 8, n. 1, pp. 3-18.

VARALDO R. (1979), Ristrutturazioni industriali e rapporti tra imprese, Franco Angeli, Milano.

VARALDO R. (2006), "Il nuovo modello competitivo aziendale dei distretti industriali", Economia e Politica Industriale, n. 1, pp. 25-42.

VARALDO R., FERRUCCI L. (1996), I distretti industriali fra logiche di sistema e logiche di impresa, Franco Angeli, Milano.

VELO D. (2007), L'Europa dei progetti. Impresa, innovazione, sviluppo, Giuffrè, Milano.

VELO D. (2011), "La varietà dei sistemi locali per l'innovazione emergente in Europa", Sinergie, n. 84, pp. 5-20.

VON STEIN J.H., ZIEGLER W. (1984), "The Prognosis and Surveillance of Risks from Commercial Credit Borrowers", Journal of Banking and Finance, vol. 8, n. 2, pp. 229-247.

ZHANG G.P., HU M.Y., PATUWO E.B., INDRO D.C. (1999), “Artificial Neural Networks in Bankruptcy Prediction: General Framework and Cross-Validation Analysis", European Journal of Operational Research, vol. 116, n. 1, pp. 16-32. 